In Crescendo, 2020; 11(1): 67-80

Fecha de recepción: 07/01/2020

Fecha de aceptación: 26/03/2020

\title{
GESTIÓN ADMINISTRATIVA EN LA CALIDAD DE ATENCIÓN EN LOS SERVICIOS DEL HOSPITAL AMAZÓNICO DE YARINACOCHA, 2019
}

\author{
ADMINISTRATIVE MANAGEMENT IN THE QUALITY OF CARE \\ IN THE SERVICES OF THE AMAZONIAN HOSPITAL OF \\ YARINACOCHA, 2019
}

Juan Nicolas Vela ${ }^{1}$

\section{RESUMEN}

La investigación que se da a conocer muestra como propósito determinar en qué medida la gestión administrativa se relaciona con la calidad de atención en los servicios del Hospital Amazónico de Yarinacocha, 2019; por lo cual, se empleó como metodología de estudio el tipo no experimental. Mediante su finalidad al estudio se le conoce como aplicada, práctica y/o empírica, mediante su carácter o profundidad es correlacional causal, mediante su naturaleza se puede afirmar que el enfoque es mixto, asimismo mediante el alcance es temporal - transversal y el tipo es descriptivo correlacional llegando al nivel explicativo. La población estuvo compuesta por 671 especialistas en salud con una muestra de 245 y como segunda población se tomó como muestra a 245 pacientes de los diversos servicios del Hospital Amazónico de Yarinacocha. Debido al tipo de tesis se empleó como instrumento de investigación cuestionarios, entrevistas y encuestas uno para cada variable; determinando la incidencia entre la gestión administrativa en la calidad de atención de los servicios del Hospital Amazónico de Yarinacocha; mediante la prueba del coeficiente Tau_b de Kendall, con una puntuación de 0,373** con una significancia de 0,000 , así también se utilizó la prueba del coeficiente de Rho de Spearman, con un valor de: $0,518 * *$, existiendo una correlación positiva moderada (media).

1 Estudiante del Programa de Doctorado en Gestión Pública y Gobernabilidad de la Escuela de Pos grado de la Universidad César Vallejo Correo Electrónico: juveva_2@hotmail.com Afiliación Institucional: Universidad Cesar Vallejo. 
Es por ello que se rechaza la hipótesis nula y se acepta la hipótesis general de estudio. Asimismo, el director de la entidad sostiene que lo más urgente en cambiar y mejorar es la infraestructura de todo el hospital, por lo cual, la parte administrativa del hospital es responsable de las gestiones de la modernización de la entidad.

PALABRAS CLAVE: Gestión administrativa, calidad de atención.

\section{ABSTRACT}

The research that is released shows the purpose of determining the extent to which administrative management is related to the quality of care in the services of the Amazon Hospital of Yarinacocha, 2019; Therefore, the non-experimental type was used as a study methodology, through its purpose the study is known as applied, practical and / or empirical, by its nature or depth it is causal correlational, by its nature it can be affirmed that the approach is mixed, also through al-cance it is temporary - transversal and the type is descriptive correlational reaching the explanatory level. The population was composed of 671 health specialists with a sample of 245 and as a second population, 245 patients from the various services of the Amazon Hospital of Yarinacocha were taken as a sample. Due to the type of thesis, questionnaires, interviews and surveys were used as research instruments for each variable; determining the incidence among administrative management in the quality of care of the services of the Amazon Hospital of Yarinacocha; using the Kendall's Tau_b coefficient test, with a score of $0.373 * *$ with a significance of 0.000 , the Spearman's Rho coefficient test was also used, with a value of: $0.518 * *$, with a positive correlation moderate (medium). That is why, the null hypothesis is rejected and the general hypothesis of study is accepted, also the director of the entity maintains that the most urgent thing to change and improve is the infrastructure of the entire hospital, therefore, the administrative part of the hospital is responsible for the modernization of the entity.

KEY WORDS: Administrative management, quality of care.

\section{INTRODUCCIÓN}

Si se habla de la gestión en administración pública, se puede mencionar que la gestión administrativa tiene como propósito tramitar y administrar las percepciones económicas que recibe el estado, con el fin de ofrecer servicios de calidad encaminados a compensar los requerimientos del residente en los ejes de: seguridad, educación, salud, etc., priorizando en todo momento las necesidades del solicitante y dando alternativas para contrarrestar la problemática de los ciudadanos, aunque por más intención de mejora, la gestión administrativa tiene diversos procesos que ocasionan un avance paulatino principalmente en el sector salud. (Sepúlveda, 2017). Los esfuerzos por cambiar la mala imagen de la gestión 
administrativa en salud pública del Perú, son cuantiosos, pero presentan insuficiencia en fortaleza y extensión, carecen de incentivos, planeación estratégica, asimismo hay escasez en el financiamiento y ni que decir al momento que se hace una licitación de los equipos y/o materiales médicos cuyos costos tienen montos exorbitantes. El trabajo es desarticulado lo que ocasiona que no existan cambios notorios ocasionando en la ciudadanía una indignación la que con lleva a exigir que el estado les brinde una mejor atención y puedan resolver sus problemas de una manera inmediata. (Aliaga, 2018).

El sector salud manifiesta múltiples carencias que influyen en una pésima gestión administrativa, por lo cual, se requiere que los trabajadores médicos y los especialistas en salud, adicionalmente a su conocimiento profesional en el área, también conozcan sobre gestión pública, porque en la gran mayoría de las entidades de salud tienen deficiencias en la gestión administrativa, específicamente en la administración de los costos debido que no tienen conocimiento del costo de atención por paciente con una enfermedad especifica; si no se tiene una cantidad exacta del costo, no se puede gestionar los recursos debido a una falta de control en los procedimientos establecidos. (Alva, 2015). Esta problemática se presenta en la mayoría de centros de salud del territorio peruano, nos centramos en esta oportunidad en el departamento de Ucayali, específicamente en el Hospital Amazónico de Yarinacocha fundado por un alemán en el año 1958, y cuya transferencia al Ministerio de Salud se hizo en el año 1980. El hospital se encuentra ubicado en la localidad de Puerto Callao en el Distrito de Yarinacocha, Provincia de Coronel Portillo.

La presente investigación se centra en el Hospital Amazónico el cual es un órgano desconcentrado y centro de referencia de la Red de Salud N² , clasificándose en base de complejidad y a características funcionales en el segundo nivel de atención, categoría II - 2, el cual, hasta el año 2017 logró una atención del 85.5 por ciento atenciones en consultas externas, atendiendo a pobladores no solo de Yarinacocha sino de Madre de Dios, Huánuco, Pasco, Loreto.

En la actualidad la cantidad de pacientes que acuden al establecimiento es el triple de lo estipulado, por lo cual, el talento humano del hospital se ve saturado debido que cuenta solo con 51 médicos, 86 enfermeras, 32 obstetras, 2 odontólogos, 98 enfermeras técnicas; ante la demanda se necesita el doble de personal como mínimo para a tender a la multitudinaria población que acude por un servicio médico. 
Además de la falta de talento humano calificado, existen diversos inconvenientes como la infraestructura, la cual tiene una antigüedad de 50 años, los ambientes son muy reducidos, se requiere una reconstrucción y en otros casos los ambientes no tienen las condiciones técnicas para la atención, también se menciona que la infraestructura del tanque elevado que proporciona agua para la cocina y más áreas tiene una antigüedad de 35 años y solo se hace mantenimiento una veces al año; todo ello suma a un alto riesgo de vulnerabilidad en la infraestructura. Asimismo, existen quejas direccionadas al personal de archivo por no brindar una buena información a los pacientes, quienes alegan que el personal administrativo en ciertos casos les duplica la información perjudicándoles a ellos porque tienen que hacer dobles pagos.

Existen múltiples casos los cuales en su conjunto conforman inconvenientes en la atención de los servicios hospitalarios, por lo cual se realizó una intensiva investigación en diversos repositorios internacionales como nacionales para extraer análisis contundentes sobre la variable independiente gestión administrativa y la variable calidad de atención, iniciando desde una perspectiva particular a la general, (local, nacional, internacional).

Desde un marco local, se estudia la investigación de Luna, (2017) "Relación entre la gestión administrativa y la percepción en el cumplimiento de los indicadores sanitarios en la Red de Salud Picota, 2017”. Tesis para obtener el grado académico de maestro en gestión de los servicios de la salud. Universidad César Vallejo. Tarapoto - Perú; donde indica que el objetivo fue identificar el vínculo entre la gestión administrativa y la percepción en el acatamiento de los indicadores sanitarios, por lo cual, se decidió trabajar con una muestra de 82 especialistas de la salud, empleando una metodología de estudio no experimental, con diseño correlacional descriptivo

Compilando las repuestas de la muestra se registra que existe en un 37 por ciento del talento humano que señala que la gestión administrativa en la Red de Salud Picota es regular, mientras que solo el 13 por ciento señala que la gestión administrativa es excelente; así también se observa que el 27 por ciento indica un nivel regular en el cumplimiento de los indicadores sanitarios y el 12 por ciento en un nivel excelente cumplieron con los indicadores en estudio. Concluyendo la investigación que existe una buena correlación entre la variable independiente gestión administrativa y la variable dependiente cumplimiento de los indicadores sanitarios, con un Chi Cuadrado de Pearson de (88.867) en la Red de Salud Picota. 
Otra conclusión fue que se debe investigar a profundidad las variables Gestión Administrativa en la Calidad de atención en los servicios de salud, se requiere analizar la teoría, por lo cual, se plasma la teoría científica de la administración, la cual estudia las funciones a ejecutarse con su respectivo lineamiento en un tiempo establecido con el propósito de buscar un incremento en el productividad inducida por el talento humano que en este caso son los médicos y administrativos de los establecimientos de salud, así como las máquinas o instrumentos que se requieren para cumplir con el servicio solicitado. La mencionada escuela de administración también es influenciada por Hobbes, quien guió para establecer los procedimientos generales la cual consiste que el talento humano siempre debe hacer lo mejor, reflejando un buen trabajo con un nivel bajo en recursos, por lo cual el desempeño laboral de cada trabajador debe ser bueno y especializarse en la función específica que le corresponda con el fin de lograr una mayor productividad con pocos recursos y en un tiempo determinado. (Taylor, 1987).

El conjunto de procedimiento, normas, principios, técnicas e instrumentos engloba al sistema del Estado peruano, el cual se divide en sistemas funcionales y administrativos. Los sistemas funcionales tienen como objetivo verificar el acatamiento de las políticas públicas, vinculadas con las capacidades y ocupaciones sectoriales; así también el sistema administrativo, tiene como objetivo regular el uso de los recursos, herramientas y/o instrumentos, así como el eficiente desempeño del talento humano. (Rodríguez, 2015).

Al referirnos a una gestión administrativa pública también se la vincula con la gestión pública por resultados, entre la población (sociedad) y un agente (gobierno), lo que implica direccionar a las instituciones estatales centrándolas en una supervisión del acatamiento de las gestiones estratégicas conceptualizadas en un propósito dirigido por el estado y un régimen de crecimiento. Aunque la coordinación sea paulatina la gestión administrativa busca un método de gestión que permita aumentar la eficacia, eficiencia y los resultados positivos en las políticas del sector estatal mediante una responsabilidad más comprometida por parte de las autoridades o funcionarios. Para mejorar la administración pública requiere considerar orientaciones de tipo empresarial, tales como outsourcing, reingeniería, benchmarking, etc., con el propósito de incrementar las capacidades y aptitudes del talento humano y máquinas y/o instrumentos de una manera más eficiente. (Bastidas \& Pisconte, 2009).

Para hablar de calidad en servicios hospitalarios, se analiza la teoría de la evolución de la teoría calidad, teniendo como definición la preponderancia o 
perfección de cierto beneficio por lo cual, el discernimiento de la cronología de la calidad inicia de la incertidumbre de una persona por alguna circunstancia. (Penacho 2000. p. 2) La gestión de calidad empieza desde un primer momento en la sección institucional en los años 1970, teniendo como finalidad principal incrementar la demanda para lograr un control del servicio, producto o del bien; en un segundo momento la calidad sigue expandiéndose con el propósito de evadir los bienes y/o productos que resulten con problemas, fallas o que los servicios brindados no sean óptimos.

La evolución de la calidad se caracteriza por tres enfoques: intervención de calidad, verificación en la calidad y dirección de importancia general. (Agüero 2012. p.3). Dentro del mundo empresarial, se determina que es necesario que exista una ventaja competitiva que es sinónimo de calidad, con el propósito de incrementar la competencia entre las empresas tornándose difícil y costosa, siendo una estrategia primordial para lograr el triunfo y subsistir ante los cambios precipitados del mercado empresarial. Lograr la calidad con lleva a beneficios como: disminución de costos o erradicar los errores de las proyecciones, disminución de los precios debido que la productividad incrementa, aumentando la intervención en el mercado del mundo empresarial y facilita e incrementa el trabajo. (Parasuraman, Zetihalm, \& Berry, 1985).

Después de la problemática descrita y fundamentar con diversas teorías se plantea la siguiente pregunta iEn qué medida la gestión Administrativa se relaciona con la calidad de atención en los servicios del Hospital Amazónico de Yarinacocha, 2019?, por lo cual, se necesita mejorar la gestión administrativa dar un cambio de 180 grados, justificando la investigación teórica cuando el objetivo de la investigación es generar una concientización y una confrontación teórica de las variables en estudio, o contrastar resultados o hacer epistemología del conocimiento existente.

Asimismo se considera un estudio práctico cuando su desarrollo ayuda a resolver un problema o por lo menos propone estrategias que al aplicarse contribuirían a resolverlo y por último, se justifica metodológicamente cuando el estudio a realizar propone un nuevo método o una nueva estrategia para generar conocimiento válido y confiable. Si un estudio propone buscar nuevos métodos o técnicas para generar conocimientos, busca nuevas formas de hacer investigación, entonces podemos decir que la investigación tiene una justificación metodológica. 
Para desarrollar el estudio en curso, se precisa como objetivo general: Determinar en qué medida la gestión administrativa se relaciona con la calidad de atención en los servicios del Hospital Amazónico de Yarinacocha, 2019; así también se señala que la hipótesis general es: La gestión administrativa se relaciona en gran medida en la calidad de atención de los servicios del Hospital Amazónico de Yarinacocha, 2019.

\section{MÉTODOS}

Al hablar de la metodología, se puede decir que es el conocimiento que analiza una serie de métodos que intervienen en un estudio científico y/o doctrinal; la técnica que se empleó en el presente estudio, fue el hipotético-deductivo. Como tipo de estudio se optó por el no experimental, debido a que se ejecuta sin la necesidad de emplear a totalidad y deliberadamente las variables (Hernández, Hernández, \& Baptista, 2010).

Mediante su finalidad, al estudio se le conoce como aplicada, práctica y/o empírica, debido al nivel de intensidad de la relación entre la variable independiente (causa) sobre la variable dependiente (efecto), constatando a través de los resultados que se requiere dar una solución práctica a la problemática o circunstancia descrita en el fundamento del estudio. Mediante su carácter o profundidad, es correlacional causal debido a que tiene como propósito analizar desde los indicadores observables y dispuestos para enumerarlos desde los fenómenos empíricos y analíticos cuyos ensayos estadísticos contribuyen para una mejor comprensión de información, asimismo también tiene como fin conocer el vínculo que existe entre distintas propiedades y la contribución del mismo en el medio real.

Mediante el alcance es temporal - transversal, debido que tiene como fin indagar sobre uno o más temas en especial en un tiempo específico, analizando su comportamiento, reacción y/o alteración, debido a que un tiempo y otro es diferente e irremediable, es por ello que se elige un tiempo o periodo en especial. Según su orientación, se emplea un estudio empírico - analítico, con el propósito de exponer, declarar y anunciar los acontecimientos dados, describiendo el porqué de las acciones o condiciones de la variable. (Hernández, Hernández, \& Baptista, 2010).

La población 1, se encuentra constituida por 671 trabajadores de las diversas áreas del hospital de Yarinacocha. La Población 2 Según la distribución de la 
población, la mayor proporción se encuentra en los distritos de Callería y Yarinacocha, seguido de Padre Abad, Irazola y Campo Verde. Obteniendo como muestra 1, trabajar con solo 245 trabajadores, al igual que la muestra 2 es un tipo de muestreo no probabilístico que se basa en el juicio personal del investigador para seleccionar a los elementos de la muestra, en este caso se seleccionó a 245 los pacientes de los diversos servicios del Hospital Amazónico de Yarinacocha.

Se empleó como instrumento de medición el cuestionario, que lo integra cuatro dimensiones: planeación organización, dirección, control, haciendo un total de 40 ítems y o preguntas, el instrumento se trabajó con la escala de Likert (1) Nunca, (2) Casi nunca, (3) A veces, (4) Casi siempre, (5) Siempre. Asimismo, se empleó como instrumento un cuestionario - instrumento del modelo SERVQUAL el cual constó de 22 preguntas agrupadas en las cinco dimensiones de la calidad del servicio, con una escala de Likert: (1) Muy desacuerdo (2) En desacuerdo (3) Indeciso (4) De acuerdo (5) Muy de acuerdo.

\section{RESULTADOS}

Siguiendo el orden sistemático de los objetivos con el fin de aceptar o rechazar las hipótesis de estudio, se empleó pruebas no paramétricas, así como: la prueba del coeficiente de Tau-b de Kendall y Rho de Spearman, con el propósito de conocer el vínculo entre variables y dimensiones.

Tabla 1 Comparación de los niveles de puntuación de la variable gestión administrativa y la variable calidad de atención en el Hospital Amazónico de Yarinacocha, 2019. Se puede apreciar que el personal de salud, manifiesta que la variable gestión administrativa del Hospital Amazónico de Yarinacocha se encuentra en un nivel "bueno" con una frecuencia de 120 (49 por ciento) seguido de un nivel excelente con una frecuencia de 101 (41 por ciento), asimismo se denota que los pacientes de los diversos servicios del hospital señalan que la variable calidad de atención tiene una mayor frecuencia en el nivel "deficiente" con una puntuación de 165 (67,3 por ciento), seguido de un nivel regular con una frecuencia de 77 (31,4 por ciento).

Tabla 2 Comparación de las dimensiones de la variable gestión administrativa en el Hospital Amazónico de Yarinacocha, 2019. Mediante la opinión del talento humano especialistas en salud, se puede determinar que el nivel las dimensiones de la variable gestión administrativa, se encuentran en un nivel de "regular", primando la dimensión "dirección” con una frecuencia de 127 (51,8 
por ciento), en segundo lugar figura la dimensión "organización" con una frecuencia de 121 (49,4 por ciento), en tercera posición figura la dimensión "planeación" con una puntuación de 120 (49 por ciento) y por último la dimensión "control" con una frecuencia de 115 (46,9 por ciento) en un nivel de bueno.

Tabla 3 Comparación de las dimensiones de la variable calidad de atención en el Hospital Amazónico de Yarinacocha, 2019. De la muestra de 245 pacientes de las diversas especialidades del Hospital Amazónico Yarinacocha. Se observa que las dimensiones de la variable calidad de atención se encuentran un nivel "regular", figurado como primera dimensión por su alta puntuación la dimensión "tangibilidad" con un frecuencia de 131 (53,7 por ciento), seguido de la dimensión "empatía" con una frecuencia de 119 (48,6 por ciento), en tercer lugar figura la dimensión "capacidad de respuesta" con una frecuencia 117 (47,8 por ciento), como cuarta dimensión "confiabilidad" con una frecuencia de 117 ( 47,8 por ciento) y como ultima dimensión "seguridad" con una frecuencia de 100 (40,8 por ciento).

Tabla 4 Determinar en qué medida la gestión administrativa se relaciona con la calidad de atención en los servicios del Hospital Amazónico de Yarinacocha, 2019. De acuerdo a las variables: Gestión administrativa y Calidad de atención, se procesó las respuestas de la muestra de 245 participantes, empleando el SPSS utilizando las pruebas del coeficiente Tau b de Kendall cuyo valor es de $0,373 * *$ con una significancia de 0,000 , que es menor al $1 \%$ de significancia estándar $(\mathrm{P}<0.01)$, asimismo se empleó la prueba del coeficiente de Rho de Spearman, indicando un valor de: 0,518**, (**) dando entender en ambos resultados que hay una mayor asociación entre ambas variables, existiendo una correlación positiva modera (media). Por ello, se rechaza la hipótesis nula y se acepta la hipótesis general de estudio: La gestión administrativa incide en gran medida en la calidad de atención de los servicios del Hospital Amazónico de Yarinacocha, 2019.

\section{DISCUSIÓN}

De acuerdo a los estudios se verifica que la administración pública, tiene como propósito tramitar y administrar las percepciones económicas que recibe el estado, con el fin de ofrecer servicios de calidad encaminados a compensar los requerimientos del residente en los ejes de: seguridad, educación, salud, etc., priorizando en todo momento las necesidades del solicitante y dando alternativas para contrarrestar la problemática de los ciudadanos, aunque por más intención 
de mejora, la gestión administrativa tiene diversos procesos que ocasionan un avance paulatino principalmente en el sector salud (Sepúlveda, 2017), por lo cual, después de una exhausta evaluación se comprueba que en el sector salud de las entidades públicas, hay carencias de calidad del servicio de atención.

Esta problemática se presenta en la mayoría de centros de salud del territorio peruano, centrándonos en esta oportunidad en el departamento de Ucayali, específicamente en el Hospital Amazónico de Yarinacocha, clasificándose en base de complejidad y a características funcionales en el segundo nivel de atención, categoría II - 2 .

A través de Bastida \& Pisconte (2009) se señala la vinculación entre el poder y la función de la administración pública, la cual desagrega una serie de procedimientos para actuar políticamente, técnicamente y jurídicamente, por lo cual, de acuerdo a la entrevista que se realizó al Director del establecimiento de Salud en estudio, sostuvo que los procedimientos de gestión con mayor lentitud en el Centro de Salud, son: procedimientos de requerimiento, licitaciones, adjudicación (ingreso del recurso humano: concurso; monitoreo del trabajo del recurso humano).

Santiago (2017) sostiene que uno de los componentes más resaltantes en el éxito de una entidad pública y/o privada es la gestión administrativa, porque se enfatiza en el conocimiento, innovación y la gestión en recursos del entorno logrando una gran repercusión positiva en el usuario, ante ello, los trabajadores del Hospital Amazónico de Yarinacocha, indican que la dimensión de gestión de recursos financieros se encuentran en un nivel de bajo al igual que la dimensión de segmentación, así también se observa que la gestión de recursos humanos se encuentran en un nivel bueno, siendo contradictorio porque, el talento humano de la institución siente incomodidad debido que ellos piden, ayuda y/o convenios para seguir sus especializaciones con la finalidad de que puedan seguir creciendo profesionalmente.

La presente situación se da debido al bajo presupuesto que destina el gobierno peruano al sector salud, así también se registra que existen diversos organismos quienes velan por los recursos públicos y su correcta distribución en todos los sectores principalmente en el sector salud, afirmando la Organización Panamericana de la Salud (OPS) de los cuarenta y dos países en América Latina y El Caribe, doce de ellos no brindan atención en salud pública, debido a la situación geográfica, escasez de inversión en el sector salud y falta de medicamentos, resaltan- 
do solo a cuatro países los cuales cumplen todos los requisitos en sus centros médicos (TeleSur, 2018).

El Hospital Amazónico, hasta el año 2017, logró una atención del 85.5\% de atenciones en consultas externas, atendiendo a pobladores no solo de Yarinacocha sino de Madre de Dios, Huánuco, Pasco, Loreto; en la actualidad la cantidad de pacientes que acuden al establecimiento es el triple de lo estipulado, por lo cual, el talento humano del hospital se ve saturado debido a que cuenta solo con: 51 médicos, 86 enfermeras, 32 obstetras, 2 odontólogos, 98 enfermeras técnicas, donde se necesita el doble de personal como mínimo para atender a la multitudinaria población que acude por un servicio médico. Sobre esto, el Director del Hospital, manifiesta que a diario atienden por consulta externa a 400 pacientes y en el área de hospitalización a 150 pacientes.

Los esfuerzos por cambiar la mala imagen de la gestión administrativa en salud pública del Perú, son cuantiosos, pero presentan insuficiencia en fortaleza y extensión, carecen de incentivos, planeación estratégica, asimismo hay escasez en el financiamiento y ni que decir al momento que se hace una licitación de los equipos y/o materiales médicos cuyos costos tienen montos exorbitantes, el trabajo es desarticulado lo que ocasiona que no existan cambios notorios ocasionando en la ciudadanía una indignación la que conlleva a exigir que el estado les brinde una mejor atención y puedan resolver sus problemas de una manera inmediata. (Aliaga, 2018).

Según la teoría científica los médicos y administrativos de los establecimientos de salud, así como las máquinas o instrumentos que se requieren para cumplir con el servicio solicitado, deberían de reflejar buenos resultados con el fin de lograr una mayor productividad con pocos recursos y en un tiempo determinado (Taylor, 1987). La realidad es distinta en diversos lugares, de acuerdo a la percepción de los trabajadores del Hospital Amazónico de Yarinacocha, la variable gestión administrativa se encuentra en un nivel "bueno" con una frecuencia de 120 (49 por ciento) seguido de un nivel excelente con una frecuencia de 101 (41 por ciento).

De acuerdo a un estudio, se verifica que existen varios requerimientos para suplir una necesidad de mejora en la gestión administrativa, empezando por el incremento en la inversión para un despliegue en las potencialidades del personal en salud a través de marcos normativos y legislativos pertenecientes a la administración en salud pública, lo que con llevará a brindar una atención de calidad (Europa Press, 2018). 
Asimismo, se denota que los pacientes de los diversos servicios del hospital, señalan que la variable calidad de atención tiene una mayor frecuencia en el nivel "deficiente" con una puntuación de 165 (67,3 por ciento), seguido de un nivel regular con una frecuencia de 77 (31,4 por ciento). Es por ello que la Organización Mundial de la Salud (OMS), afirma que los países bajos tienen una inversión menor al promedio del sector salud, perjudicando al diez por ciento de sus pacientes hospitalizados porque contraen más enfermedades en los mismos centros asistenciales debido a una mala praxis, falta de higiene y condiciones básicas en atención, inclusive se afirma que un paciente en un grupo de diez fallece por una mala atención en el proceso de recuperación. (Europa Press, 2018).

De acuerdo a las variables: Gestión administrativa y Calidad de atención, se procesó las respuestas de la muestra de 245 participantes, empleando el SPSS utilizando las pruebas del coeficiente Tau b de Kendall cuyo valor es de $0,373 *$ con una significancia de 0,000 , que es menor al $1 \%$ de significancia estándar $(\mathrm{P}<0.01)$, asimismo se empleó la prueba del coeficiente de Rho de Spearman, indicando un valor de: 0,518**, (**)dando entender en ambos resultados que hay una mayor asociación entre ambas variables, existiendo una correlación positiva modera (media). Por ello, se rechaza la hipótesis nula y se acepta la hipótesis general de estudio: La gestión administrativa incide en gran medida en la calidad de atención de los servicios del Hospital Amazónico de Yarinacocha, 2019.

Asimismo, se analiza en el estudio de Sánchez \& Solórzano, (2017) quienes indican que la atención de salud es un proceso continuo y complejo, que lograr que la atención en el servicio hospitalario sea óptima es difícil pero no imposible, obteniendo como resultados que el 88 por ciento de pacientes internados no habían tenido inconvenientes en la admisión al nosocomio, mientras que el 37 por ciento afirma que durante el proceso de hospitalización tuvieron problemas en la atención, calificando en un 68,42 por ciento en un nivel de muy mala atención, debido a la falta de comunicación directa en el proceso de hospitalización, ocasionando que afecte al paciente internado, permaneciendo el mismo 8 días más a lo establecido por el médico. Así también, se verifica que los pacientes del Hospital de Yarinacocha señalan en un nivel bajo la atención inmediata. 


\section{CONCLUSIONES}

$>$ Se determinó la relación entre la gestión administrativa en la calidad de atención de los servicios del Hospital Amazónico de Yarinacocha; mediante la prueba del coeficiente Tau_b de Kendall, con una puntuación de 0,373** con una significancia de 0,000 , así también se utilizó la prueba del coeficiente de Rho de Spearman, con un valor de: 0,518**, existiendo una correlación positiva moderada (media). Es por ello que, se rechaza la hipótesis nula y se acepta la hipótesis general de estudio.

$>$ Se determinó la relación entre la dimensión planeación en la calidad de atención de los servicios del Hospital Amazónico de Yarinacocha; mediante la prueba del coeficiente Tau_b de Kendall, con una puntuación de 0,371** con una significancia de 0,000 , así también se utilizó la prueba del coeficiente de Rho de Spearman, con un valor de: 0,507**, existiendo una correlación positiva moderada (media). Es por ello que, se rechaza la hipótesis nula y se acepta la hipótesis general de estudio.

Se determinó la relación entre la dimensión organización en la calidad de atención de los servicios del Hospital Amazónico de Yarinacocha; mediante la prueba del coeficiente Tau_b de Kendall, con una puntuación de 0,332** con una significancia de 0,000 , así también se utilizó la prueba del coeficiente de Rho de Spearman, con un valor de: 0,460**, existiendo una correlación positiva moderada (media). Es por ello que, se rechaza la hipótesis nula y se acepta la hipótesis general de estudio.

> Se determinó la relación entre la dimensión dirección en la calidad de atención de los servicios del Hospital Amazónico de Yarinacocha; mediante la prueba del coeficiente Tau b de Kendall, con una puntuación de 0,365** con una significancia de 0,000 , así también se utilizó la prueba del coeficiente de Rho de Spearman, con un valor de: 0,496**, existiendo una correlación positiva moderada (media). Es por ello que, se rechaza la hipótesis nula y se acepta la hipótesis general de estudio.

- Se determinó la relación entre la dimensión control en la calidad de atención de los servicios del Hospital Amazónico de Yarinacocha; mediante la prueba del coeficiente Tau b de Kendall, con una puntuación de 0,317** con una significancia de 0,000, así también se utilizó la prueba del coeficiente de Rho de Spearman, con un valor de: 0,443**, existiendo una correlación positiva moderada (media). Es por ello que, se rechaza la hipótesis nula y se acepta la hipótesis general de estudio. 


\section{REFERENCIAS BIBLIOGRÁFICAS}

Aliaga, R., (2018) "iPor qué es tan caótico el sistema de salud peruano? Actualidad / Salud. 26 de enero. Conexión Esan. Perú. Recopilado en: https://www.esan.edu.pe/conexion/actualidad/ 2018/01/26/por-que-es-tan-caotico-el-sistema-de-salud-peruano/

Alva, G., (2015) "La gestión de instituciones de salud en el Perú". Actualidad / Salud. 16 de junio. Conexión Esan. Perú. Recopilado en: https://www.esan.edu.pe/conexion/actualidad/ 2015/06/16/gestion-instituciones-salud-siglo-xxi-peru/

Agüero, H. (2013) Gestión de la Calidad. Recuperado de: https://goo.gl/weFN1U

Bastidas D., \& Pisconte J., (2009) “Gestión Pública”. Material de Trabajo. Programa de formación: Desarrollo de Capacidades para el fortalecimiento de las organizaciones políticas. Mayo. International. IDEA Institute For Democracy and Electoral Assistance. Recopilado en: http://www2.congreso.gob.pe/sicr/cendocbib/con4_uibd.nsf/8453BD9D9F57489405257C 0C0014A7FC/\$FILE/Gesti\%C3\%B3n_P\%C3\%BAblica.pdf

Hernández, R., Fernández, C., \& Baptista, P. (2010). Metodología de la Investigación. México D.F.: Mcgraw-HILL/Interamericana Editores, S.A. de C.V

Luna J., (2017) "Relación entre la gestión administrativa y la percepción en el cumplimiento de los indicadores sanitarios en la Red de Salud Picota, 2017”. Tesis para obtener el grado académico de maestro en gestión de los servicios de la salud. Universidad César Vallejo. Tarapoto - Perú.

Parasuraman, A., Zeithaml, V., \& Berry, L. (1985). A conceptual model of service quality and its implications for future research. Journal of Marketing, 49, 41-50. Recuperado de https:/www.researchgate.net/publication/225083670_A_Conceptual_Model_of_Service_ Quality_and_its_Implication_for_Future_Research_SERVQUAL

Penacho j. (2000). Evolución de la historia de la calidad en el contexto del mundo de la empresa y el trabajo. Obtenido de evolución de la historia de la calidad en el contexto del mundo de la empresa y el trabajo. Recuperado de: https://goo.gl/yHAv4i.

Rodríguez F., (2015) "Gestión de Capacitación en Gestión Pública". Proyecto Asegurando el Agua y los Medios de Vida en la Montaña. Junio. Instituto de Montaña. Usaid From The American People. Ancash - Perú. Recopilado en: http://mountain.pe/recursos/attachments/article/ 163/38.\%20Manual\%20de\%20gestion\%20publica.pdf

Sepulveda, A., (2017) "Estos son los problemas de la Administración a la hora de innovar" 18 de noviembre. Retina. El País. Madrid - España. Recopilado en: https://retina.elpais.com/ retina/2017/11/06/tendencias/1509957380_625096.html

Taylor, F. (1987) "Principios de la Administración”. Científica. El Ateneo, Bogotá. 